\title{
Optimization Algorithms of Objective Control of Technical Objects
}

\author{
Genady Burov, Riga Technical University
}

\begin{abstract}
During the process of design, industrial production and operation of technical objects significant amount of information is accumulated. It can be used for the development of mathematical algorithms and programs for realization of automated computer control of technical objects. Because of the complexities in mathematical formalization of the aprioristic information difficulties arise, which do not always allow using it actively in mathematical models for the estimation of parameters obtained at separate stages of processing of the numerical information about measured transients. As the formed mathematical models are based on the theory of technical object identification, there is dependence of the computing stability of algorithms. Therefore, it is necessary to use equation systems with reasonably low orders. Such objective can be achieved due to the application of filtration of decomposition of autoregressive function. The algorithm of the objective control of technical objects is offered in the form of an algorithm by using the theory of pattern recognition. The recognition models as part of control algorithm may be built on the base of Fourier model. This results in efficiency of algorithms especially in test procedures.
\end{abstract}

Keywords - Characteristic polynomial, decomposition, discrete transfer function, regressive model, technical object transfer function.

\section{ANALYSIS OF ALGORITHM OF THE SOLUTION OF DIFFERENCE OF EQUATIONS}

On the basis of measurements of signals of the technical object description of them can be developed in the form of difference equations (DE), which have been obtained during the design phase in the form of transfer functions (TF) (into the Laplace domain $W(p)$ ). Obviously, this is a task for a mathematical model, which converts the digital measurements of signals at the input and output of the technical object into quantitative values of coefficients of its transfer function (TF) $W(p)$. The model of theoretical identification solves this task by solving a system of difference equations [3], [14]:

$$
\begin{aligned}
& X \bar{\alpha}+Y \bar{\xi}=\bar{y} \\
& U=[X Y] ; \\
& U \bar{\theta}=\bar{y} \quad \bar{\theta}=U^{-1} \bar{y}=\left[\begin{array}{l}
\bar{\alpha} \\
\bar{\xi}
\end{array}\right]
\end{aligned}
$$

Vector $\bar{\theta}$ connects the values of the input and output signals at specific time intervals and with factors of technical object TF $W(p)$. However, it consists of abstract values. The mathematical description of this connection is given in [13]. Thus, the expression of generating operator of the system (1) can be derived:

$$
\begin{aligned}
& D(z)=\frac{H(z)}{B(z)}=\frac{y(z)}{x(z)} \\
& x(z)=\Phi z(T) x(p) \\
& y(z)=\Phi z(T) y(p)
\end{aligned}
$$

Here $\Phi z(T)$ denotes the quantization operation, known as operation Z-transform [2], [13]. It is implemented by decomposition (3) into the sum of elementary fractions and by applying Z-transform to each summand:

$$
\begin{aligned}
& D(z)=\Phi z(T) W(p) \\
& D(z)=\frac{H(z)}{B(z)}=\sum_{i=1}^{n} \frac{A w_{i} z}{z-\exp \left(-a w_{i} T\right)} \\
& A w_{i}=\frac{R w\left(p=-a w_{i}\right)}{\coprod_{j=1(j \neq i)}^{n}\left(a w_{i}-a w_{j}\right)} .
\end{aligned}
$$

As it can be seen from equations

$$
\begin{aligned}
& \Phi z(T) Q w(p) \Rightarrow B(z) ; \\
& B(z)=\prod_{i=1}^{n}\left(z-\beta_{i}\right) \\
& Q w(p)=\prod_{i=1}^{n}\left(p+a w_{i}\right) \\
& \beta_{i}=\exp \left(-a w_{i} T\right) \\
& \bar{\theta} \Rightarrow \bar{w} \quad \bar{w} \in W(p) \\
& W(p)=\frac{R w(p)}{Q w(p)}
\end{aligned}
$$$$
Q w(p)=\prod_{i=1}^{n}\left(p+a w_{i}\right)=\sum_{i=0}^{n} q_{i} p^{i}
$$

Let us consider an opportunity of realization of algorithm

We use operation inverse Z-of return transformation, applying expressions (3)-(7) upside-down: 


$$
\begin{aligned}
& \Phi z^{-1}(T)\left\{\widehat{D}(z)=\frac{\widehat{R}(z)}{\widehat{B}(z)}\right\} \Rightarrow \hat{W}(p) \\
& \bar{\alpha} \Rightarrow \widehat{R}(z) \quad \bar{\xi} \Rightarrow \widehat{B}(z)
\end{aligned}
$$

The above polynomials are formed separately on the basis of the vector coefficients $\bar{\alpha}$ and $\bar{\xi}$, in the vector $\bar{\theta}$ composition. Therefore, for model (8) evaluation it is necessary to analyze the nature of computing operations (3)-(7). We must start by the characterizing polynomial (10) common denominator root definition

$$
\widehat{B}(z) \Rightarrow \prod_{i=1}^{n}\left(z-\widehat{\beta}_{i}\right)
$$

This analysis has shown that the individual root obtaining operation is imperative for any standardized computing algorithm. It may be impossible in the case of compressed information (impossible to distinguish between the calculated roots). At baseline analog poles for operator $W(p)$ were well differentiated, as they were on the left plane of complex numbers and the distance between them was large enough. However, according to (7), these roots moved to the right complex plane in the restricted area at reduced distances. If the root finding operation cannot be carried out accurately, the next operation - the distribution of the vector $\bar{D}_{M}(z)$ sum of elementary fractions (9) and (10) cannot be carried out. It is concluded from the formula

$$
V_{i}=\frac{\hat{R}\left(z=\widehat{\beta}_{i}\right)}{\prod_{j=1 ; j \neq i}^{n}\left(\widehat{\beta}_{j}-\widehat{\beta}_{i}\right)} .
$$

If for discrete pole splitting the Taylor series is one we obtain

$$
\begin{aligned}
& \prod_{i=1(i \neq j)}^{n}\left(\beta_{i}-\beta_{i}\right)=T^{n-1} \cdot \prod_{i=1(i \neq j)}^{n}\left(a_{j}-a_{i}\right) \\
& V_{i} \geq \frac{1}{T^{n-1}} \cdot \frac{\hat{R}\left(z=a_{i}\right)}{\prod_{j=1 ; i \neq i}^{n}\left(a_{j}-a_{j}\right)} .
\end{aligned}
$$

Further it is necessary to establish $\widehat{D}(z)$ in (9) as a sum

$$
\widehat{D}(z) \Rightarrow \sum_{i=1}^{n} \frac{V_{i} z}{z-\beta_{i}}
$$

and to transform the summarized parts by using formula (7). These are nonlinear discrete-pole $\beta_{i}$ operations for turning into analogues $\vartheta_{i}$

$$
\left[\beta_{i}=\exp \left(-T \vartheta_{i}\right)\right] \Rightarrow\left[\vartheta_{i}=\frac{\ln \left(\beta_{i}\right)}{T}\right] \text {. }
$$

Using the formula

$$
\Phi z^{-1}(T)\left[\frac{V_{i} z}{z-\beta_{i}}\right] \Rightarrow \frac{V_{i}}{p-\vartheta_{i}}
$$

expression (22) turns into the form

$$
\begin{aligned}
& \Phi z^{-1}(T) \hat{D}(z) \Rightarrow\left\{\hat{W}(p)=\sum_{i=1}^{n} \frac{V_{i}}{p-\vartheta_{i}}\right\} \\
& \widehat{W}(p)=\frac{\hat{R} w(p)}{\widehat{Q} w(p)} .
\end{aligned}
$$

Therefore, model (8) computing operations are mathematically incorrect.

Abstract vector $\bar{\theta}$ in (2) cannot modify parameters that characterize the physical state of the object

$$
\Phi z(T) \bar{\theta} \Rightarrow \hat{W}(p) \text {. }
$$

\section{OPTIMIZATION OF ALGORITHM OF THE DECISION DIFFERENCE EQUATIONS}

The system of the equations (2) contains a methodical error. It is designated as $Y x(p)$

$$
y(p)=Y w(p)+Y x(p)
$$

We use decomposition

$$
\begin{aligned}
& y(p)=x(p) \cdot W(p)=\sum_{i=1}^{m} \frac{A x_{i}}{p+a x_{i}}+ \\
& a x_{i} \in x(p) ; a w_{i} \in W(p) \\
& y(p)=\frac{R(p)}{Q^{(n)}(p)}=\sum_{i=1}^{n} \frac{A_{i}}{\left(p+a_{i}\right)} \\
& y(t)=\sum_{i=1}^{n} A_{i} \cdot \exp \left(-a_{i} t\right) .
\end{aligned}
$$

We use operations (4)-(7)

$$
\begin{aligned}
& \Phi z(T)\left\{y(p)=\frac{R(p)}{Q^{(n)}(p)}\right\} \Rightarrow y(z) \\
& y(z)=\frac{R(z)}{B(z)} \\
& \Phi z(T)\left[Q^{(n)}(p)=\prod_{i=1}^{n}\left(p+a_{i}\right)\right]=B(z) \\
& B(z)=\prod_{i=1}^{n}\left(z-\beta_{i}\right)=\sum_{i=0}^{n} \xi_{i} z^{n-i} ;
\end{aligned}
$$


$2014 / 53$

$$
\beta_{i}=\exp \left(-a_{i} \cdot T\right)
$$

Vector $\bar{w}^{T}(t)$ of values $y(t)$ on an interval

$$
\bar{t}^{(n)}=\left[\begin{array}{lllll}
t & t+T & t+2 T & \cdots & t+n T
\end{array}\right]
$$

can be written down as the sum of lines of the matrix

$$
[H]_{i j}=A_{i} \cdot \exp \left(a_{i} t\right) \cdot \beta_{i}{ }^{j-1} .
$$

Let us consider function of autoregression [15]

$$
\begin{aligned}
& \left.u(t)=y(t)+y[t-T)] \cdot \xi_{n-1}+y[t-2 T)\right] \cdot \xi_{n-2} \cdots+ \\
& +y[t-n T)] \cdot \xi_{0}
\end{aligned}
$$

Here $\xi_{i}$ are factors of transfer function of the filter

$$
\begin{aligned}
& B \xi(z)=z^{n}+z^{n-1} \cdot \xi_{n-1}+ \\
& +z^{n-2} \cdot \xi_{n-2}+\cdots+z^{n-k} \xi_{n-k}
\end{aligned}
$$

According to (30) expression (28) leads to a kind

$$
\begin{aligned}
& H=D(\overline{d g}) G ; \quad \overline{d g}_{i}=A_{i} \cdot \exp \left(-a_{i} T\right) \\
& G_{i j}=\beta_{i}{ }^{j}
\end{aligned}
$$

Here the diagonal matrix is submitted by the vector of factors $\overline{d g}$. Then for $\mathrm{k}=4$ we shall receive

$$
\begin{gathered}
H \bar{\xi}=D g\left[\begin{array}{l}
A_{1} \exp \left(a_{1} t\right) \\
A_{2} \exp \left(a_{2} t\right) \\
A_{3} \exp \left(a_{3} t\right) \\
A_{4} \exp \left(a_{4} t\right)
\end{array}\right] \cdot\left[\begin{array}{l}
B \xi\left(z=\beta_{1}\right) \\
B \xi\left(z=\beta_{2}\right) \\
B \xi\left(z=\beta_{3}\right) \\
B \xi\left(z=\beta_{4}\right)
\end{array}\right] \\
{\left[\begin{array}{l}
B \xi\left(z=\beta_{1}\right) \\
B \xi\left(z=\beta_{2}\right) \\
B \xi\left(z=\beta_{3}\right) \\
B \xi\left(z=\beta_{4}\right)
\end{array}\right]=\left[\begin{array}{lllll}
1 & \beta_{1} & \beta_{1}{ }^{2} & \beta_{1}{ }^{3} & \beta_{1}{ }^{4} \\
1 & \beta_{2} & \beta_{2}{ }^{2} & \beta_{2}{ }^{3} & \beta_{2}{ }^{4} \\
1 & \beta_{3} & \beta_{3}{ }^{2} & \beta_{3}{ }^{3} & \beta_{3}{ }^{4} \\
1 & \beta_{4} & \beta_{4}{ }^{2} & \beta_{4}{ }^{3} & \beta_{4}{ }^{4}
\end{array}\right] \cdot\left[\begin{array}{l}
\xi_{4} \\
\xi_{3} \\
\xi_{2} \\
\xi_{1} \\
\xi_{0}
\end{array}\right]}
\end{gathered}
$$

or in a brief form

$$
\begin{aligned}
& H \bar{\xi}=\overline{d g} \otimes \overline{B \xi}=\left[\begin{array}{c}
A_{1} \exp \left(a_{1} t\right) \\
A_{2} \exp \left(a_{2} t\right) \\
A_{3} \exp \left(a_{3} t\right) \\
A_{4} \exp \left(a_{4} t\right)
\end{array}\right] \overline{\exp \left(a_{i} t\right)} \otimes C \\
& H \bar{\xi}=\overline{\exp \left(a_{i} t\right)} \otimes \bar{C} ; \quad \bar{C}=\bar{A} \otimes \overline{B \xi} \\
& B \xi_{i}=\prod_{j=1}^{n}\left(\beta_{i}-\mu_{j}\right)
\end{aligned}
$$

$$
\begin{aligned}
& F(\overline{\xi x}) y(t)=\sum_{i=1}^{n} C_{i} \cdot \exp \left(-a_{i} t\right) \\
& C_{i}=A_{i} B \xi_{i} \quad C_{i}=A_{i} \prod_{j=1}^{n}\left(\beta_{i}-\mu_{j}\right) .
\end{aligned}
$$

Using the received expressions for (29) it is received

$$
\begin{aligned}
& u \xi(t)=\sum_{i=1}^{n} C_{i} \cdot \exp \left(a_{i} t\right) \\
& C_{i}=A_{i} B \xi\left(z=\beta_{i}\right) .
\end{aligned}
$$

According to (32) the matrix (2) will be transformed to a kind

$$
U \overline{\xi x} \Rightarrow\left\lfloor X=0 ; Y^{*}=Y \overline{\xi x}\right\rfloor .
$$

The transformed system of the equations is joint concerning a vector $\overline{\xi w}$

$$
Y \overline{\xi_{w}}=0 \quad \overline{\xi w} \in B w(z)
$$

The new model of algorithm does not contain the methodical error. Conditionality of the matrix is better because of the decrease in its order.

\section{OPTIMIZATION OF ALGORITHMS OF THE OBJECTIVE CONTROL OVER FOURIER MODELS}

Restoration TF $W(p)$ on algorithm (8) is connected to significant errors. The reason is that the roots of the analogue characteristic polynomial $Q w(p)$ denominator of the TF $W(p)$ move from the left part of the infinite plane of complex numbers to its right part, to a narrow region of the right positive semicircle. With the decrease of $\mathrm{T}$, they contract in one multiple unit root. Therefore, large errors are characteristic of the recovery operations of discrete roots with the coefficients of the polynomial $B(z)$ due to the constraints of the allocation of individual roots, since the distance between them can be compared to errors of computerized rounding of numbers. Therefore model (8) cannot be implemented because of the information compression of fragments $W(p)$ due to sampling (4).

$$
\begin{aligned}
& \bar{\theta} \Rightarrow[\hat{D}(z)=\hat{H}(z) / \hat{B}(z)] \\
& \Phi z^{-1}(T) \bar{\theta} \Rightarrow \hat{W}(p)
\end{aligned}
$$

The results of computations in (4) are non-observable in nature. It indicates that for the identification of the dynamic characteristics of technical objects, it is appropriate to apply methods of pattern recognition theory rather than direct analytic transformations. Using the former, the measurements of the observed features are mapped into the measurements of nonobservable patterns. For technical objects the task is simplified because the relationship between the features and patterns can 
always be described using mathematical equations. It follows from the Weierstrass Theorem, according to which such a description can be obtained in the form of approximating polynomials [6]. In this case, the relationship is described by the Laplacian pattern

$$
y(p)=W(p) x(p) .
$$

According to equation (12), for fixed test input $x(p)$, the matrix of vectors $\overline{y_{i}}$ of values of transient processes $M y$ at intervals of fixed length is calculated. The relationship between matrices of vectors in the model

$$
M=M w \cup M y
$$

is described by the convolution equation. Therefore, the mapping of the set of vectors of features $M y$ onto the set of vectors of patterns $M w$ is isomorphism. The model of the objective control (MOC) is based on mapping

$$
M w \Leftrightarrow M y .
$$

The main task of the pattern recognition theory classification of features and patterns in (13) - is fulfilled a priori [3] on the basis of isomorphism (14). According to the Weierstrass Theorem it is always possible to find mathematical description of the relationship between the elements not included in matrix (13) $\bar{w}_{i} \notin M w, \bar{y}_{i} \notin M y$ in the form of an approximating polynomial. Therefore, the decision function for the implementation of the transient process in the model of objective control may be formed in an analytical form to restore the numerical value of coefficient vector $W_{i}(p)$ by the numerical value of the vector of transient process

$$
\bar{y}_{i} \Rightarrow \bar{w}_{i} ; \quad \bar{w}_{i} \in M w, \quad \bar{y}_{i} \in M y .
$$

In [7] it is indicated that the decision function can be approximated by a function close to the linear one, by using the operation of endomorphism (by mapping models - matrices (13) "onto themselves"), in which their vectors can be decomposed into isomorphic basic systems, $B z w^{(n)}$ and $B z y^{(n)}$ . They are formed on the basis of variations (12) in increments different from that in (13). The operation of endomorphism maps models in (13) onto the space of spectral vectors of the Fourier expansion of matrix elements (13) in the basic system

$$
\begin{aligned}
& \operatorname{Fur}\left(B z y^{(n)}\right) M y^{(m)} \Rightarrow C y^{(m)} \\
& \operatorname{Fur}\left(B z w^{(n)}\right) M w^{(m)} \Rightarrow C w^{(m)} \\
& \operatorname{Fur}\left(B z y^{(n)} ; B z w^{(n)}\right)(M y \bigcup M w) \Rightarrow \\
& \Rightarrow\left(C y^{(m)} \bigcup C w^{(m)}\right)
\end{aligned}
$$

Isomorphism (14) is transformed into the isomorphism of the transformed model

$$
\begin{aligned}
& (M w \Leftrightarrow M y) \rightarrow\left(C w^{(m)} \Leftrightarrow C y^{(m)}\right) \\
& {[M y w=M y \bigcup M w] \Rightarrow[C y w=(C y \bigcup C w)] .}
\end{aligned}
$$

The corresponding algorithm is based on the operation of inversion of Gram matrices

$$
\begin{aligned}
& G r w=\left(B z w^{T} B z w\right) \\
& G r y=\left(B z y^{T} B z y\right) ; \\
& \bar{w}_{i}=B z w \cdot G r y^{-1}\left(B z y^{T} \bar{y}_{i}\right) .
\end{aligned}
$$

To form the model (13), expression $W(p)$ of the transfer function of the object is required; it is derived from the DE system, which includes differential equations in the operator form (22). Coefficients of characteristic polynomial $Q w(p)$ include the weight of the object.

The error of the OCM can be measured using the stationarity of changes of the vector of spectral coefficient. The nonlinear properties of operators of mapping deform the isometric properties of working spaces and are a source of methodical errors. Formally they represent a certain dynamic process generated by $y(p)=W_{i}(p) x(p)$ on the interval t0. According to (16) and (17) it is received

$$
\begin{aligned}
& C y_{i}=(G r y)^{-1}\left\lfloor B z y^{T} \bar{y}_{i}(t)\right\rfloor \\
& C w_{i}=(G r w)^{-1}\left\lfloor B z w^{T} \bar{w}_{i}(t)\right\rfloor \\
& G r y=B z y^{T} B z y \\
& G r w=B z w^{T} B z w \\
& \sigma_{i}=\sqrt{\frac{1}{N} \sum_{i=1}^{N}\left\|\overline{C y}_{i}-\overline{C y}_{0}\right\|} \\
& N=\left(t_{2}-t_{1}\right) / T .
\end{aligned}
$$

This leads to the conclusion that for the estimation of algorithm usability characteristics of stationary of Fourier systems can be used. The accuracy of algorithm can be measured on the basis of the best approximation. For this purpose, let us use more accurate characteristics than the one used in [7]. It consists in the measurement of projections of vectors $\bar{y}_{i}(t)$ and $\bar{w}_{i}$ on the Fourier bases. Using the matrices of projection, we get

$$
\begin{aligned}
& U=B z y^{T}[G r y]^{-1} B z y^{T} \\
& S=B z w^{T}[G r w]^{-1} B z w^{T} .
\end{aligned}
$$

Then the deviations of vectors from the subspaces of bases are:

$$
u_{i}=(I-U) \bar{y}_{i}
$$


$2014 / 53$

$$
s_{i}=(I-S) \bar{w}_{i}
$$

From here we get angular values of deviations:

$$
\begin{aligned}
& \varphi y_{i}=\operatorname{arctg}\left(\frac{\left\|\left(I-U_{i}\right) \bar{y}_{i}\right\|}{\left\|U \bar{y}_{i}\right\|}\right) \\
& \varphi w_{i}=\operatorname{arctg}\left(\frac{\left\|\left(I-S_{i}\right) \bar{w}_{i}\right\|}{\left\|S_{i} \bar{w}_{i}\right\|}\right) .
\end{aligned}
$$

We shall note that the isometric properties of OCM algorithm can be received by the estimation

$$
\Delta \varphi_{i}=\varphi y_{i}-\varphi w_{i}
$$

These phase values and their discrepancy characterizes the isometric properties of the operator of mapping. The error of the OCM can be measured using the stationarity of changes of the vector of spectral coefficient. The nonlinear properties of operators of mapping deform the isometric properties of working spaces and are a source of methodical errors. Formally they represent a certain dynamic process generated by $y(p)=W_{i}(p) x(p)$ on the interval $t 0$. According to (16) and (17) it is received

$$
\begin{aligned}
& C y_{i}=(G r y)^{-1}\left[B z y^{T} \bar{y}_{i}(t)\right\rfloor \\
& C w_{i}=(G r w)^{-1}\left\lfloor B z w^{T} \bar{w}_{i}(t)\right\rfloor \\
& G r y=B z y^{T} B z y \quad G r w=B z w^{T} B z w \\
& \sigma_{i}=\sqrt{\frac{1}{N} \sum_{i=1}^{N}\left\|\overline{C y}_{i}-\overline{C y}_{0}\right\|} .
\end{aligned}
$$

\section{EXAMPLE}

Below the results of a numerical experiment are presented which verify the accuracy to use OCM for identification of parameters of an object with $\mathrm{TF}$

$$
W(p)=\frac{p+\theta}{p^{3}+q_{2} p^{2}+q_{1} p+q_{0}} .
$$

Vectors $\bar{w}_{i} \in M w$ were given by deviations from the nominal value

$$
\bar{w}_{0}^{T}=\left[\theta_{0} ; q_{00} ; q_{10} ; q_{20}\right]=[0.5 ; 4 ; 1.863 ; 0.537]
$$

in increments $\varepsilon=0.05 ; \quad \bar{w}_{i}=\bar{w}_{0}(1+\varepsilon i)$; in interval $\left.i \in \overline{1.5}\right)$. The formation of OCM begins with the calculation of matrices for model (13). The accuracy of OCM (see $\varepsilon q(\%)$ ) remained high. Due to the compression of information, an insoluble task to restore $W(p)$, formula (10) was solved on the basis OCM.
Here the damped sinusoid had a frequency of $2.5 \mathrm{Gz}$, the object

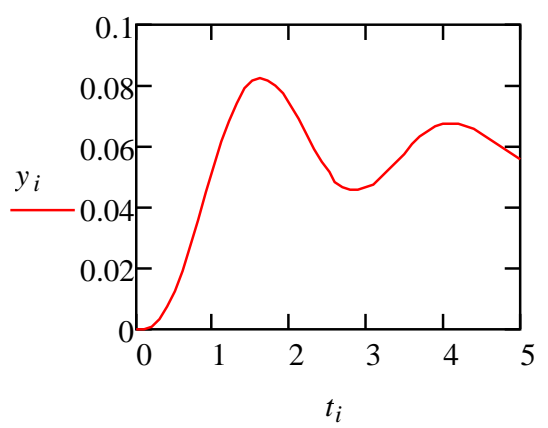

reaction $y(t)$ is presented in Fig. 1 .

Fig. 1. The object reaction $y(t)$.

Basic matrices Bzq, Bzy (16) and $M y(Y x)$ were calculated as well as the matrix of transient processes $M w \Rightarrow M y$ was calculated by formula $y(p)=W(p) x(p)$. Basic systems were used:

$$
\begin{aligned}
B z y & :=\left(\begin{array}{ccc}
0 & 0 & 0 \\
0.00024 & 0.00024 & 0.00024 \\
0.0033 & 0.00327 & 0.00324 \\
0.02258 & 0.02229 & 0.02203 \\
0.12385 & 0.12201 & 0.12037 \\
0.6259 & 0.61589 & 0.60703
\end{array}\right) \\
B z q & :=\left(\begin{array}{ccc}
4 & 3.45535 & 3.00526 \\
10.6 & 10.04989 & 9.55372 \\
6.4 & 6.20952 & 6.03636 \\
1 & 1 & 1
\end{array}\right)
\end{aligned}
$$

To check the accuracy of OCM, a matrix

$$
M w^{(j)}(Q x) \in W^{(i)}(p)
$$

was developed

$$
M w(Q x):=\left(\begin{array}{ccccc}
4 & 2.772 & 2 & 1.489 & 1.139 \\
10.6 & 9.279 & 8.249 & 7.424 & 6.749 \\
6.4 & 5.94 & 5.575 & 5.278 & 5.032 \\
1 & 1 & 1 & 1 & 1
\end{array}\right) .
$$

The accuracy of its recovery from the matrix of transient processes $M y(Y x)$ was verified. Matrix $M w(Q x)$ was identified by matrix $M y(Y x)$ of the transient processes: 


$$
M y(Y x):=\left(\begin{array}{ccccc}
0 & 0 & 0 & 0 & 0 \\
2.39 \cdot 10^{-4} & 2.36 \cdot 10^{-4} & 2.33 \cdot 10^{-4} & 2.31 \cdot 10^{-4} & 2.30 \cdot 10^{-4} \\
3.30 \cdot 10^{-3} & 3.23 \cdot 10^{-3} & 3.17 \cdot 10^{-3} & 3.12 \cdot 10^{-3} & 3.08 \cdot 10^{-3} \\
0.023 & 0.022 & 0.021 & 0.021 & 0.021 \\
0.124 & 0.119 & 0.116 & 0.114 & 0.112 \\
0.626 & 0.602 & 0.584 & 0.572 & 0.56
\end{array}\right)
$$

Matrices of spectral vectors $[C y]$ and $[C q]$ were also calculated (16)

$$
\begin{aligned}
\text { Cy } & :=\left(\begin{array}{ccccc}
0.40709 & -1.02713 & -1.77714 & -2.18136 & -2.39909 \\
1.3513 & 1.6911 & 1.38367 & 0.84219 & 0.23442 \\
-0.7597 & 0.33527 & 1.39125 & 2.33466 & 3.15762
\end{array}\right) \\
\mathrm{Cq} & :=\left(\begin{array}{ccccc}
1 & 0.358 & 3.879 & 9.049 & 14.726 \\
4.657 \times 10^{-10} & -1.31 & -10.806 & -23.362 & -36.685 \\
1.164 \times 10^{-10} & 1.951 & 7.927 & 15.313 & 22.957
\end{array}\right) .
\end{aligned}
$$

The matrix was identified

$$
\mathrm{qx} 0:=\left(\begin{array}{ccccc}
4.01447 & 2.74241 & 1.85357 & 1.20089 & 0.70314 \\
10.63759 & 9.31091 & 8.35966 & 7.6462 & 7.09264 \\
6.41046 & 5.95113 & 5.61632 & 5.36175 & 5.16204 \\
0.99869 & 0.99924 & 0.99778 & 0.99549 & 0.99296
\end{array}\right)
$$

The accuracy of the algorithm OCM (\%) is demonstrated in the matrix $\varepsilon q(\%)$

$$
\begin{gathered}
\varepsilon q^{(j)}=\left\{\left\lfloor M w^{(j)}(Q x)-q x 0^{(j)}\right\rfloor / M w^{(j)}(Q x)\right\} 100 \% \\
\varepsilon q(\%):=\left(\begin{array}{ccccc}
0.00362 & -0.01075 & -0.07304 & -0.19372 & -0.38268 \\
0.00355 & 0.00347 & 0.01342 & 0.02992 & 0.05098 \\
0.00164 & 0.0019 & 0.00748 & 0.01593 & 0.02593 \\
-0.00131 & -0.00076 & -0.00222 & -0.00451 & -0.00704
\end{array}\right)
\end{gathered}
$$

Gram matrix is close to singular

$$
\operatorname{det}(\text { Gry })=7.85 \times 10^{-21} .
$$

Therefore, it is possible to implement the OCM only using special software [10], [12].

\section{CONCLUSION}

Identification models based on conventional arithmetic equation solutions for signal measurements cannot be used for objective control. The arithmetic equation vectors $\bar{\theta}$ are abstract. Their relation to physical parameters can be quantified in analytical relationship. However, there are mathematically incorrect operations and results are not transparent. The operational computer software of automatic control and diagnostic systems of vector $\bar{\theta}$ decrypting and the development of technical objects are doomed to failure. For the in-depth diagnosis of the object technical state, new methods must be used. Control models must include image recognition models that allow incorrect mathematical operations to provide the right solutions. The implementation of the image recognition system for Fourier models depends on the form of the basic information, which is not always sufficient and does not enable escape from significant errors.

\section{REFERENCES}

[1] G. Burovs, D. Varslavs, and M. Buikis, "Finite Difference Models of Identification of Dynamic Processes," Scientific Proc. of Riga Technical University, Technologies of Computer Control, vol. 5, pp. 66-76, 2001.

[2] M. Buikis, and G. Burov, "Forecasting properties of recurrent relation," Scientific Proc. of Riga Technical University, Technologies of Computer Control, vol. 5, 2002.

[3] G. Burov. 2002. "Structural disbalancement of regression models of identification," Scientific Proc. of Riga Technical University in series Computer Science, Boundary field problems and computer simulation, vol. 44, 2002

[4] J. Grundspenkis, and G. Burov, "The analysis of topological characteristics of information identification models," Scientific Proc. of Riga Technical University in series Computer Science, Applied Computer Systems, vol. 4, 2001.

[5] J. Grundspeṇkis, and G. Burovs, "Topological properties of recurrent relations in models of identification," Scientific Proc. of Riga Technical University in series Computer Science, Applied Computer Systems, vol. 5, 2002.

[6] G. Burovs, "Imitācijas modelu izmantošana parametru kontroles algoritmos," Pārskats ZPD NAA VPSN-02/02-2013, pp. 7-17, 2013.

[7] G. Burov, "The Computer Diagnosing of Technical Objects with the Use of Pattern Recognition Principles," Scientific Journal of Riga Technical University, Boundary field problems and computer simulation, vol. 52, 2013.

[8] G. Burovs, "Tehnisko objektu datorizēta diagnosticēšana, izmantojot attēlu atpazī̌šanas principus," Pārskats ZPD NAA VPSN-02/02-2013, pp. $18-43,2013$.

[9] M. Buikis, and G. Burovs, "Matemātiska un programmu nodrošināšana datorizēto sistēmu diagnosticēšanas algoritmiem," Pārskats ZPD NAA VPSN-02/02-2013, pp. 49-57, 2013.

[10] G. Burov, "Combinatorial methods of formation of parallel algorithms of the signals processing," Scientific Proc. of Riga Technical University in series Computer Science, Boundary field problems and computer simulation, vol. 45, 2003.

[11] G. Burov, "Principles of formation of parallel algorithms of the information processing in dynamic objects," Scientific Proc. of Riga Technical University in series Computer Science, Technologies of computer control, vol. 3, 2003.

[12] G. Burovs, "Symbolical Combinatory Model of Parallel Algorithm of Identification That Uses Method of Least Squares," Scientific Proc. of Riga Technical University in series Computer Science, Boundary field problems and computer simulation, vol. 37, 2008.

[13] J. M. Smith, Mathematical modelling and digital simulation for engineers and scientists. New York, Chichester: Wiley, 1980.

[14] D. Graupe, Identification of Systems. New York: IEEE Press, 1976.

[15] G. E. P. Box, G. M. Jenkins, Time Series Analysis: Forecasting and control. USA, University of Wisconsin: Holden-Day, 1970.

Genady Burov was born 1937 in St. Petersburg, Russia. He received the Doctoral degree in 1966. Genady Burov is currently a Leading Researcher with the Environment Modeling Center of Riga Technical University.

E-mail: emc@cs.rtu.lv 